\title{
Health Care Leadership and Management-The Changing Face, Making Difference
}

${ }^{1}$ Gaurav Thukral, ${ }^{2}$ Vishesh Madaan

\begin{abstract}
Health care organizations are the most intricate and exacting to manage because of the diverse field and dynamic innovative changes and spiraling costs. In this article, we discuss different approaches to leadership in health care and that whatever approaches chosen should endeavor for a strong team identity, inspiring vision, and strong commitment to collaborating teamwork. Framework of process management established in three domains-align, enable, and innovate-is also discussed. Many bodies of research have focused on the competencies related to effective managerial/leadership skills. Guidance for effective leadership should focus on the communication, relationship management, motivational leadership, collaboration, business skills, and knowledge. Effective leaders provide highquality empathetic patient care. They prioritize patient satisfaction by ensuring their voice is heard. We must strive toward collective and consistent leadership by clinicians and other staff at the forefront, rather their designated managers alone.
\end{abstract}

Keywords: Competencies, Health care organizations, Leadership approaches, Patient satisfaction, Process management.

How to cite this article: Thukral G, Madaan V. Health Care Leadership and Management-The Changing Face, Making Difference. Curr Trends Diagn Treat 2017;1(2):107-110.

Source of support: Nil

Conflict of interest: None

\section{INTRODUCTION}

Health care sector has become increasingly intricate, medical professionals need to develop skills to demonstrate outcomes sophisticated enough for this advancing and ever-innovating field. Evidence-based medicine is the new mantra, and several efforts are being made all over to define the competencies most relevant for health care. ${ }^{1}$

Management experts assume that a well-functioning organization in any field is akin to a well-oiled machine, with good output efficiency. This can hold true only when work

${ }^{1}$ Executive Vice President and Chief Operating Officer, ${ }^{2}$ Business Analyst

${ }^{1}$ Health Care at Home India Private Limited, Noida, Uttar Pradesh, India

${ }^{2}$ Department of Strategy, Home India Private Limited, Noida Uttar Pradesh, India

Corresponding Author: Gaurav Thukral, Executive Vice President and Chief Operating Officer, Health Care at Home India Private Limited, Noida, Uttar Pradesh, India, e-mail: gaurav. thukral@hcah.in is stated in detail and compartmentalized to well-defined operational units. Clinicians often object to the elaborate specifications, and managers lament a lack of coordination. ${ }^{2}$

A good leader directs the activity of his teammates toward a shared goal. The key quality of a leader is to influence his group in a positive manner and help his team to cope up with ever-changing scenarios. Most of the theories on leadership in the health care were developed for the business setting and later applied in health sector context, which itself is a dilemma for its application. ${ }^{3}$

A consortium of six major professional membership organizations have pointed out five competency domains common among all practicing health care managers. They used the research and experience of their individual credential processes to arrive at these five domains:

1. Communication and relationship management,

2. Professionalism,

3. Leadership,

4. Knowledge of the health care system,

5. Business skills and knowledge.

Leaders need to engage to characterize knowledge, skills, and abilities within each domain and to find out which of these competencies were common among members of the Health care Leadership Alliance and which were specific to the members. ${ }^{1}$

It was observed by Drucker ${ }^{3}$ that health care organizations are the most complex in human history and that it is difficult to manage even a small health care institute. Few years have passed since this observation, but the complexities of health care organizations and its leaders and managers have not diminished in any manner. Leaders and managers are expected to do a lot with minimal resources at their disposal.

There are several theories on leadership and these are dynamic as they keep changing according to the changing times. The early Great Man Theory states that some people have an inherent quality and characteristics to be good leaders. Common leadership styles have been described earlier by various behavioral theories as authoritarian, democratic, and laissez-faire. ${ }^{4}$

Intricacy of health care system is unmatched because of the compulsions of several disease areas, different goals, and staff of numerous disciplines. Health care systems are formed of different professional groups, departments, and specialties having complex, nonlinear 
interactions. The interactions might be supportive or be in conflict with each other. This diversity in such organizations need to be capitalized upon by leaders, that is, they need to capitalize the resources and increase the efficiency of the system. The highly intricate environment system of the health care sector can be taken care of by adapting to a number of leadership approaches. ${ }^{5}$

Health care leaders have a moral responsibility to promote organizational ethical environment. Health care provider's moral distress and positive ethical climate are both connected to the system's ability to retain health care professionals and enhance their job satisfaction. We recommend that leaders should provide access to ethics education and resources, offer interventions, such as ethics debriefings, establish ethics committees, and/or hire a bioethicist to develop ethics capacity and to assist with addressing health care provider's moral distress. ${ }^{6}$

\section{LEADERSHIP APPROACHES}

A number of leadership approaches can be adapted to the health care setting to optimize management in this highly complex environment.

\section{Transformational Leadership}

The leader needs to focus on the worth of teammates, especially if they have a common sense of mission. It moves beyond the old concept of traditional style of transactional leadership. They need to communicate their vision and goals in more effective, meaningful, and creative way and empower others. These leaders transform the organizations and can achieve results far beyond expectations. Transformational leaders are able to motivate performance beyond expectations through their ability to influence attitudes., 7

\section{Collaborative Leadership}

This type of leadership works toward a cooperative process. This leadership is about aligning individual work toward sharing knowledge and experience, and achieving their goals. Collaborative health leadership requires a coordinated work atmosphere where multiple partners are encouraged to work together as a team while implementing effective practices and processes. This promotes understanding of different work cultures, enhances efforts at integration, and nurtures interdependency between different health care stakeholders. This requires a synergistic work environment where different disciplines work as a unit to implement effective processes. ${ }^{7,8}$

\section{Transactional Leadership}

This type of leadership in organizations plays an exchange role between leaders and the rest of the team mates. The leaders motivate followers by exchange, like giving rewards to teammates on completing some goals. The focus of the leader is to accomplish a given task or achieving a goal, and the exchange might be a reward or punishment for the same. ${ }^{9}$

\section{Democratic Leadership}

These leaders involve most of the team players in decision-making process, they encourage group work, and team players are highly engaged and responsible in a project, which encourages creativities. But it matters when quick decision-making and efficiency are required. ${ }^{10}$

\section{Autocratic Leadership}

In this leadership, the power lies with the leader and team players have hardly any opportunity to make decisions. This kind of leadership is highly efficient, and decisionmaking is very quick and implementation is immediate. But the disadvantage is that the group dynamics are lacking and the staff feels out of place. ${ }^{10}$

\section{Laissez-Faire Leadership}

This type of leader lacks direct supervision of the staff. This hinders appropriate feedback to the employees. Highly trained and specialized employees form a part of laissez-faire leadership. This approach to leadership obstructs the working of staff that requires supervision and feedback. This type of leadership can be a bane or boon to the organization. Teammates are welcome to share views and suggestions, but it might lead to poor productivity and lack of control in the presence of unskilled staff. This type of leadership is also known as hands-off style. Leader has little control on the teammates and gives them independence to carry out their tasks. $^{11}$

\section{Basic Framework for Process Management}

The organizations establish their management processes in three basic domains, namely align, enable, and improve as shown in the illustration. This model was developed by the institute for enterprise excellence and adapted for health care value. The three domains are as follows:

Align: The leaders comprehend a vision and establish critical strategies in achieving that vision. They discern the essential metrics and then convey the direction on a regular basis. The astuteness of the frontline workers finally drives the alignment process.

Enable: Here, the leaders visit the work areas to get a perception of the daily tasks and challenges. They invigorate the people to develop and tame the hurdles which hinder the process of change. They also help people 
to accept failures as learning experiences and celebrate their achievements.

Improve: Leaders continuously learn by observing and translate these observations to enable frontline teams to develop new care models that can upgrade the patient experience. For achieving a better patient experience, we should abstain from a top-down approach and rather enable the frontline caregivers to render the patients what they actually need and appreciate. This approach can improve the daily functioning of the health care system. $^{12}$

A framework on process management was developed by the Institute for Enterprise Excellence and adapted for health care settings by Theda Care Centre for Health care value. The organization ground their management of process in three basic areas.

Achieving a better patient experience means eschewing top-down objectives and instead empowering frontline caregivers to give patients what they say they actually value. In this way, we can improve the daily functioning of the health care system, one institution at a time (Fig. 1). ${ }^{12}$

\section{Identifying Common Competencies}

The analysis of the qualifications and certification process of the management members was done on a large scale which disclosed a number of interrelated supportive competencies. The task led to these knowledge, skills and capabilities getting positioned together into five competency domains that were similar among the membership of all six associations: ${ }^{1}$

1. Communication and relationship management: The capability to communicate clearly and briefly

Principles

Create value for the

흥 strategies to achieve that

vision. Set high but

reasonable targets.

Communicate the direction on a regular basis

Motivate, mentor inspire Energize people to develop and overcome barriers to change. Daily be in the work area to listen

to understand. Embrace

failure, celebrate success

Breakthrough thinking Continuously learn by listening, seeing and translating observations. support new models fo care delivery developed by front line

with internal and external customers to create and preserve relationships and to make possible smooth constructive interaction with individuals and groups.

2. Leadership: The capability to motivate individual and organizational excellence, obtaining common future strategic vision by managing successfully the changes to attain organizations' outstanding performance.

3. The capability to collaborate personal and organizational conduct with ethical and professional standards that helps patients as well as community and providing lifelong commitment to improve learning.

4. Knowledge of health care environment: The clear understanding and interpretation of health care system and the environment under which health care officials and managers function.

5. Business skills and knowledge: The quality of applying business skills and principles to the health care environment for improved functioning in financial management, human resource, information, risk, quality management, organizational dynamics and governance, and strategic planning and marketing.

Aforementioned five domains were viewed as the common competencies with current focus on outcomes and evidence-based management.

\section{Achieving Patient Satisfaction}

The ultimate goal of any health care service is to benefit the patient and the community as a whole. Therefore, managers and team leaders must ensure good patient relations and satisfaction. All staff members should be trained to respect patient's rights. They should provide clear and

Management

Organizing and translating Establish a structure to achieve the plan. Organize and allocated resources. Monitor structure to ensure consistency and alignment to plan

Empower, involve and coach

Empower authority within parameters of area to improve and solve problems break-down silos by involving cross-functional teams to solve value stream issues. Coach problem solving daily

Monitor and maintain predictability Monitor the outputs of each system to ensure stability and a standard outcome continuously challenge the process to identify areas of improvement

\section{Frontline}

Sitting and achieving goals identify meaningful goals that can be accomplished in their area that directly affect the overall vision and strategy daily report on status and needed support

Develop and share Be self-developer. find opportunities to grow and develop to better support the organization share with other what is working and what is not working

Adapt and adjust Adapt the tools by making incremental adjustment that all shifts agree with. Treat tools as a countermeasure not a solution. Structurally solve area problems daily

Fig. 1: Basic framework for process management 
precise information to the patients so that they can take informed decisions regarding treatment options and lifestyle modifications to improve their health conditions.

Patient's satisfaction should be assessed by using appropriate and valid tools by health care centers for two-way feedback system. This will include

- Client satisfaction survey

- Suggestion boxes

- Community consultation committees

Positive attitude of staff toward the patient will influence their willingness to follow the instructions, complete the prescribed treatment, and continue health care. ${ }^{13}$

\section{Result-oriented Health Care Leadership}

A clinician faces a number of challenges in the intricate setting of health care. There is constant change in societal needs, patient expectations, new techniques, and spiraling costs of treatment. It is for the clinician to gauge the situation, work toward the benefits of patients and community at large to equally distribute the resources available to produce quality clinical care and improvement in the patients' well-being.

The result oriented leadership in health care needs to focus on delivering the desired results and meeting the needs under three areas, namely individuals, team and tasks. A leader must facilitate effective and efficient health care provision, since results in the system are the crucial point in establishing the success of the organization. ${ }^{14,15}$

A man who fails to plan, plans to fail. Therefore, in an organization, planning is of utmost importance for success, moreover enough time should be given to deal with an unplanned event and to communicate effectively with staff, patients, and community. A manager should include following information in his/her planner.

- Important dates: Time period for yearly patient satisfaction survey, time for supervisory visits, and time for community health meetings.

- Managing information: Managing routine patientmonitoring report, progress reports, and important meetings with partners.

- Managing finances: Budget preparation and financially monitoring assets.

- Managing hardware: Hardware inspection and maintenance dates, ordering deadlines for supplies and hardware. ${ }^{13}$

\section{CONCLUSION}

Many theories, cases, and models have influenced the current leadership strategies that can be applied to the health care setting. Guidance for effective leadership should focus on the dynamic relationships between leadership values, culture, capabilities, and the organizational context. The leader's developmental journey must operate within this dynamic, supported by a high level of self, team, and organizational awareness. Leadership development has clearly reached a critical crossroad, and the most important role of the leader could be described as ensuring a ready supply of replacement leaders to maintain organizational progress in the ever-changing health care environment.

\section{REFERENCES}

1. Stefl ME. Common competencies for all healthcare managers: the Healthcare Leadership Alliance model. J Healthc Manag 2008 Nov-Dec;53(6):360-374.

2. Plesk PE. Complexity, leadership, and management in healthcare organisations. BMJ 2001 Sep;323:746.

3. Drucker P. Managing in the next society. Common competencies for all healthcare managers. New York: Truman Talley Books, St. Martin's Griffin; 2002.

4. Al-Sawai A. Leadership of healthcare professionals: where do we stand? Oman Med J 2013 Jul;28(4):285-287.

5. Bolman LG, Deal TE. Reframing leadership. Business leadership. San Francisco (CA): Jossey-Bass; 2003. pp. 86-110.

6. Bell J, Breslin JM. Healthcare provider moral distress as a leadership challenge. JONAS Healthc Law Ethics Regul 2008 Oct-Dec;10(4):94-97, quiz 98-99.

7. Atchison TA, Bujak JS. Leading transformational change: the physician-executive partnership. Chicago (IL): Health Administration Press; 2001.

8. Harrison B. The Nature of leadership: historical perspectives \& the future. J Calif Law Enforcement 1999;33(1):24-30.

9. Burns JM. Leadership. New York: Harper and Row; 1978.

10. Amanchukwu RN, Stanley GJ, Ololube NP. A review of leadership theories, principles and styles and their relevance to educational management. Management 2015;5(1):6-14.

11. Bass BM, Avolio, BJ. The implications of transactional and transformational leadership for individual team and organizational development. In: Woodman RW, Pasmore WA, editors. Research in organizational change and development. Vol. 4. Greenwich (CT): JAI Press; 1990. pp. 231-272.

12. Toussaint JS. How healthcare systems can effectively manage process. Waltham (MA): NEJM Catalyst; 2016. [cited 2016 Jun 20]. Available from: http://catalyst.nejm.org/how-healthsystems-can-effectively-manage-process /.

13. Anonyms. Chapter 10: leadership and management. 2016. [cited 2016 Apr 6]. Available from: http://www.who.int/ hiv/pub/imai/om_10_leadership_management.pdf?.

14. Al-Touby SS. Functional results-oriented healthcare leadership: a novel leadership model. Oman Med J 2012 Mar;27(2):104-107.

15. Almgren G. Health care politics, policy, and services: a social justice analysis. 2nd ed. New York (NY): Springer Publishing Company; 2012. [cited 2012 Mar 26]. Available from: https:/ / www.ncbi.nlm.nih.gov/pubmed/18727749. 